\title{
A Review On The Diverse Application Of Silver Nanoparticle
}

\author{
V. Thamilselvi and K.V. Radha* \\ Bioproducts Lab, Department of Chemical Engineering, A.C. Tech, Anna University, \\ Chennai 600025, India
}

\begin{abstract}
Nanotechnology is an emerging field with diverse applications in pharmacy, medicine, industries and environments. Among nanosized particles, the inorganic nanoparticles posses unique physical and chemical properties. These nanoparticles and their composites are useful for the wide range of applications. This review aims to provide a consolidated assessment of silver nanoparticle and silver nanocomposites in various fields. Among nanoparticles, silver nanoparticle exhibits antimicrobial activities and prominent in diverse applications. The nanosilver composites by coating of chemical material, agricultural residues, other matrices etc., have been used for the development of new materials that could be conveniently used in house cleaning solutions, fabric cleaners, wound bandages, paints, ornamental glasses, electronics circuits, fridge, washing machines and cosmetics, etc., Similarly, silver nanoparticle can also be functionalized with various groups of commercial adsorbents and agricultural residues to increase their affinity towards target compounds and used as a selective sorbents for metal ions and anions.
\end{abstract}

Keywords: Nanotechnology, Nanocomposites, Antimicrobial, Agricultural residues, Adsorbents, Affinity, Silver nanoparticle, Target compounds

\section{INTRODUCTION}

The use of silver nanoparticle (AgNP) in various fields is receiving attention among researchers in recent years. The physical, chemical and mechanical properties of AgNP are unique and useful in the field of electronics and electrical, biotechnology and Bioengineering, textile engineering, environmental and pharmaceuticals. The metal nanoparticles have surface Plasmon resonance (SPR) and its strong observation of light is utilized for fabricating the optical devices [1]. The SPR has depended on the oscillations of the free electrons of the particles and its surrounding medium and form the conduction band due to the electromagnetic field [2]. The geometrical and spectral characteristics of silver nanoparticle, such as size, shape, interparticle spacing and the environment provide important control on linear and nonlinear optical properties [3]. The optical sintering effects, the blue and red shift of AgNP also utilized in the field of optical sensors [4]. The excitation of the plasmon (SPR) enhances the electromagnetic field in the surrounding environment and produce measurable changes in nanoparticle that response in the optical field. The light scattering property of AgNP is also utilized for the detection of heavy metal (lead, arsenic etc.,) impurities in water [5]. The physical and chemical properties of nanoparticle were unique and differ from their bulk properties [6]. The plasmonic properties of $\mathrm{AgNP}$ that emphasize the sensitivity of the extinction spectra of supporting substrates like silica microsphere and indium tin oxide coated glass slide [7]. In analytical chemistry AgNP is widely used for its selectivity and sensitivity as sensors. The increasing and decreasing size of the nanoparticles influences the light radiation on the SPR band that was utilized for developing analytical sensor devices [8]. Other than the optical, mechanical properties of silver nanoparticle are involved in the catalytic activity which was influenced by the size, concentration, synthesis methodology and its supporting medium [9]. This review article outlined the research advances of silver nanoparticle in optical, mechanical and catalytic properties and their applications in various fields.

\section{OPTICAL APPLICATION OF SILVER NANOPARTICLE AND SILVER NANOCOMPOSITES}

The optical properties of the silver nanoparticle are owing to the interaction between incoming light and free conduction electrons. When the wavelength of the incident light matches with the oscillating frequency of the conduction electron, a surface plasmon resonance occurs, this gives rise to the absorption band in the visible region. This surface plasmon resonance peak depends on the particle size, shape, surface charge, separation between the particle and the nature of the environment. This leads to the formation of different colors of the metal nano-sol. The surface structure of the nanoparticles determines the charge of the nanoparticles. The optical property of AgNP was examined with various experiments and they depend on their size, shape and surrounding medium. For example the IBANS (intensely and broadly absorbing nanoparticles) are used for light harvesting applications [10]. The SPR devices are used in a variety of chemical and biological sensor systems. The SPR characteristics of silver nanofilm coated on the SAM (Self Assembled Monolayers) substrate and their 
interplay was determined by SPP (Surface plasmon polarization) and LSP (Localized Surface Plasmon) oscillations in the multilayer system and utilized for various analytical purposes. The silver nanoparticle electrodes are been reported as a sensor for the urine samples to detect the presence of $\mathrm{Cl}^{-1}$ ions [11].

The silver nanoparticle are used in solar cells for trapping solar energy. In conventional solar cells the coating layer was made up of silicon. So the system was not efficient because silicon is a poor absorber of light. Hence the light trapping efficiency of solar cells was enhanced by undercoating the silver nanoparticle layer along with silicon layer. The dielectric layer of silver nanoparticle increases the refractive index of silicon from $490 \mathrm{~nm}$ to $1050 \mathrm{~nm}$ [12]. Similarly the silver nanoparticle film on Poly-methylmethacrylate-Poly ethylene terephthalate membrane (Ag-PMMA-PET), the composite has been used for ultra thin light filters [13]. In optical fiber analyzers the Ag-doped silica nanocomposite is coated as a membrane on the surface of an optical fiber along with bent silica. The improved optical sensor fiber, useful to trace ammonia in a gas sample [14].

The plasmonic and photonic characteristics of silver nanoparticle and the silver nanoparticle loaded compound, such as silver loaded poly vinyl alcohol (Ag-PVA) (Figure 1) are used for light guiding and optical sensing applications $[15,16]$. Silver nanoparticle are useful in semiconductor applications due to its rapid trapping of free electrons. In semiconductors $\mathrm{TiO}_{2}$ are the known photocatalyts and their efficiency is improved by capping with silver nanoparticle. Hence the semiconductor with the photocatalyst of $\mathrm{TiO}_{2}$-AgNP nanocomposites is used for better semiconductor efficiency [17].

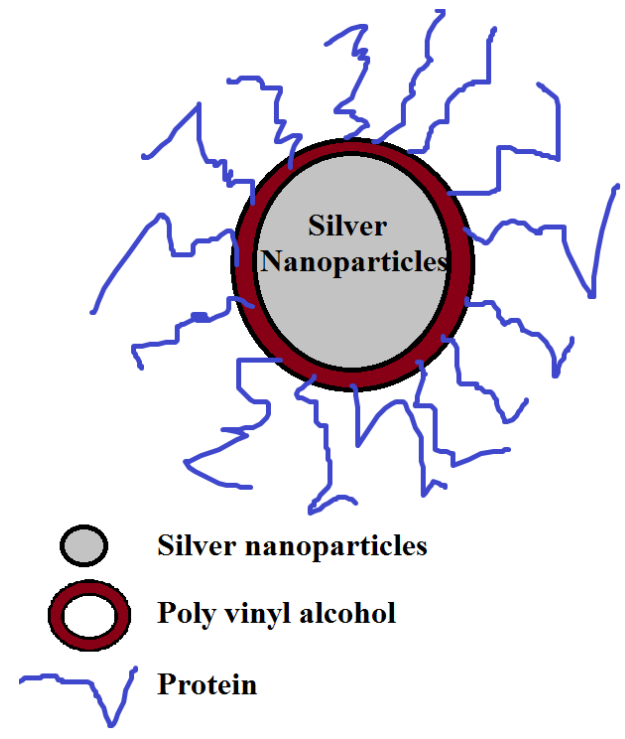

Figure 1Ag-PVA composite acts as a sensor for detecting protein

The silver nanoparticle composites are used in optical enzyme biosensor for sugar analysis. Such biosensors are made with the stimuli-responsive hydrogel-silver nanoparticle composite, constructed by immobilizing in a glucose oxidase (GOx). When a sample solution such as glucose was applied to the surface of this optical enzyme biosensor, the interparticle distances of the silver nanoparticle present in the stimuliresponsive hydrogel were increased, and thus the absorbance strength of the LSPR was decreased. The concentration of glucose is determined by using the optical enzyme biosensors [18].

\section{ELECTRICALS AND ELECTRONICS APPLICATIONS OF SILVER NANOPARTICLE AND SILVER NANOCOMPOSITES}

Silver nanoparticle is successfully employed in the field of microelectronic materials. The melting point of smaller sized silver nanoparticle drastically reduced and with increased surface energy. This property of silver nanoparticle is useful in electronics and used as conductive fillers in electronically conductive adhesives (ECAs) [19]. The electrical conductors fabricated with a thick film of silver nanoparticle reduces the electrical loss. The lower electrical losses at higher frequency is attributed to the lower surface roughness that give better packing and used to fabricate antennas [20]. The electro reflectance (ER) effect of silver nanoparticle is important in the field of electro -optical devices and sensors. In this case, the change in the electronic charge which stored on the particles that alters the absorption spectrum of the particle ensemble. The effect is typically 100 times stronger than for a bulk metal surface, and is readily discernible to the unaided eye. The ER effect makes it possible to directly monitor the changes in the electrostatic charge stored on small metal particles using absorption spectroscopy [21]. 
The nanocomposites of dielectric Teflon matrix embedded with silver nanoparticle have increased electrical conductivity with increased film thickeness at various silver nanoparticle concentration [22]. Now a day multiwalled carbon nanotube (MWNTs) are used as electrodes that possess high electrical and mechanical properties. The electrical conductivity significantly improved using silver (Ag)/polymer composites by incorporating multiwalled carbon nanotubes (MWNTs) decorated with self-assembled nano-Ag particles. The conductivity achieved for the composite $\left(2.5 \times 10^{5} \mathrm{~S} / \mathrm{cm}\right)$ was even higher than that of bulk tungsten $\left(1.9 \times 10^{5} \mathrm{~S} / \mathrm{cm}\right)$, and used in the fields of interconnects and micropackaging systems in electrical devices [23].

\section{BIOENGINEERING AND BIOMEDICAL APPLICATIONS OF SILVER NANOPARTICLE AND SILVER NANOCOMPOSITES}

Nanoparticle in medicine are used for therapeutic, imaging, and diagnostics of cancer and other diseases leading an entrapped or bound therapeutic or diagnostic target material in the area of interest, e.g., a tumor. The destination of targeted delivery may be found by physical forces (magnetic) or with surface bound antibodies. The unique plasmonic and easy surface chemistry for a variety of functionalization with silver nanoparticle are used for biomedical imaging and photothermal theraphy. The cytotoxicity and cellular uptake nature of engineered silver nanoparticle with carbohydrates are significantly improved such as modified with glucose, lactose and oligosaccharides. These carbohydrate modified AgNP may provide new tools that can be used in phototherapy for killing cancerous cells and diagnosis [24]. Silver nanoparticle possesses a strong fluorescence signal that is utilized for the identification of calf thymus DNA ( $c t$-DNA) [25]. In electrochemical DNA sensors, silver nanoparticle are used as a indicator that bounded with the oligonucleotide probes that able to paired with the sample DNA sequence for disease detection application such as disease diagnosis, drug screening, epidemic prevention and environmental protection. Silver nanoparticle are used in variety of techniques including fluorescent, radiochemical, piezoelectrio surface plasmon resonance spectroscopy and quartz crystal microbalance. The electrochemical detection for sequence analysis, depends on the surface plasmon resonance and piezoelectric technology, hense silver nanoparticle probes are used.

AgNPs have been used as a significant tool for the anticancer treatment. Silver nanoparticle are acts as a nanocarriers, significant tool for the anticancer treatment. Silver nanoparticle coated on a biocompatible surface, based on polyethylene glycol through a modification of the emulsion solvent-evaporation technique, adopted for the site specific cancer treatment. In vitro observations on the cervical cancer-derived cell lines HeLa and CaSki, using flow cytometry studies at $24 \mathrm{~h}$, employing propidium iodide (PI) and carboxyfluorescein diacetate succinimidyl ester (CFSE) as reporter molecules. Both free and encapsulated AgNPs were toxic for both cell lines, inducing important decrements on the cell viability compared with cisplatin -an anticancer chemotherapy drug [26].

The interaction between nanomaterials and biological systems exploits in the field of biomedical engineering. Recently, nanoparticle PCR research gained more attention and their effects along with titanium dioxide is evaluated using real-time PCR machine [27]. The anti-inflammatory property of AgNP also exploited in mouse models in their pro-inflammatory cytokine systems that inhibits interferon gamma activity and tumor necrosis factor alpha. Hence silver nanoparticle plays a important role in anti-inflammatory therapies [28,29].

The redox property of silver nanoparticle is useful for the successful preparation of DNA sensors and the electrochemical polymerization of conducting polymer -pyroll that coated with silver nanoparticle possesses good sensitivity and stability [30]. Silver nanoparticle conjugated to oligonucleotides have recently emerged as powerful tools for the detection of target DNA sequences, and are used in the design of colorimetric assays based on aggregation induced by sequence-specific hybridization [31]. The Figure 2 showed the site targeted identification of the desired probe using the detector DNA probe tagged with silver nanoparticle [32]. This DNA-sensing concept used to detect the targeted DNA that can be captured in a sandwich DNA hybrid assay.

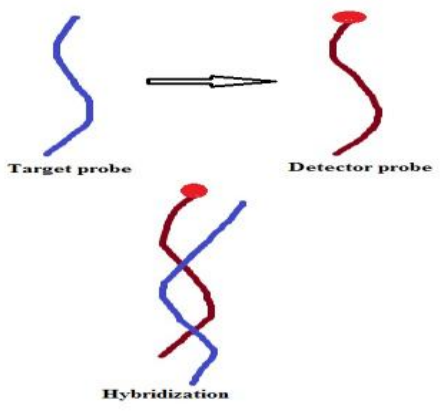

Figure 2 Site targeted analysis using detector DNA probe tagged with silver nanoparticle 
The emerging field of nanomedicine seeks to exploit the novel properties of engineered nanomaterials for diagnostics and therapeutic applications. The engineered nanoparticles are used to carry drug payloads, image contrast agents or gene therapeutics for diagnosing and treatment. Silver nanoparticle are being used increasingly in wound dressings, catheters, and various household products due to their antimicrobial activity. The chitin-AgNP composite scaffolds are widely used in tissue engineering applications. These scaffolds have good blood clotting ability and are used for wound healing applications [33]. In tissue engineering silver nanoparticle loaded on poly 3-hydroxybutyrate co-3-hydroxyvalerate (PHBV) nanofibers scaffolds, are significant in antibacterial activity and invitro cell compatibility. These bioengineered scaffolds are potentially used for joint arthroplasty [34]. The biodegradable Poly L-lactide (PLA) ultrafine fibers loaded with silver nanoparticle are used as implanted medical devices like urinary catheters. The electrospinning technology is used for fabricating these PLA-AgNP scaffolds and used for wound healing or anti-adhesion membranes [35]. Polymethyl methacrylate loaded with nanosilver is being considered as bone cement and the nanosilver can improve the antimicrobial activity [36]. Ultrahigh molecular weight polyethylene fabricated with silver nanoparticle are used in joint replacement components, the silver nanoparticle that reduces the wear and tear of the polyethylene polymer [37]. The surgical meshes that made of silver nanoparticle coated polypropylene are used as an ideal candidate for surgical meshes due to its antimicrobial, anti-inflammatory properties [38].

\section{TEXTILE INDUSTRIAL APPLICATION OF SILVERNANOPARTICLE AND SILVER NANOCOMPOSITES}

Silver nanoparticle deposited onto the surface of different fabrics (nylon, polyester and cotton) by ultrasound irradiation, possess the antibacterial activity. The coated fabrics have potential application in sports socks to control odour, dirts, microbes, etc., and used in medical bandages and bed linings as shown in Figure 3 [39].

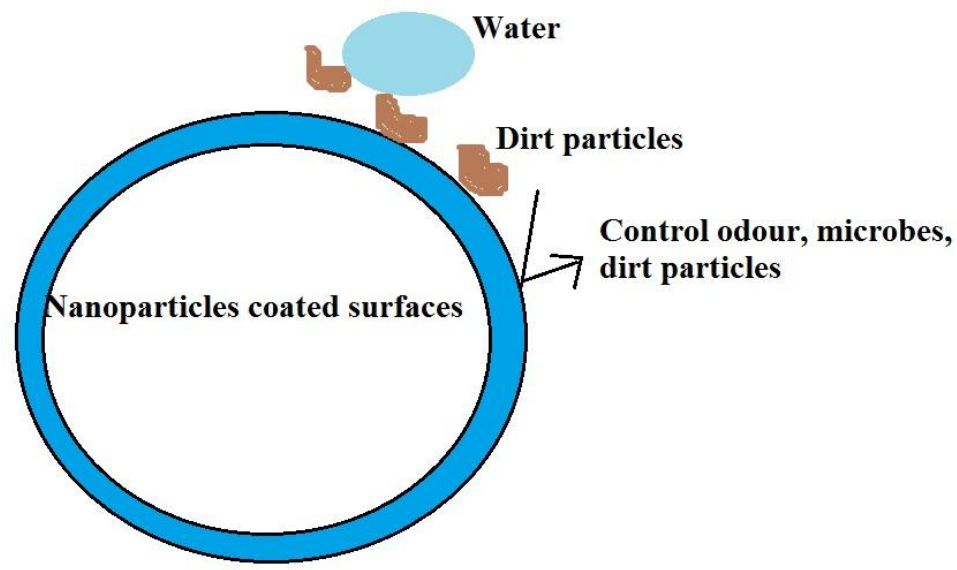

Figure 3 Nanoparticles coated fabrics resistant to dirt particles, odour and microbes

The chemical functionalization of silver nanoparticle with chemicals or their incorporation in suitable polymer matrices used to produce a polymeric nanoparticle composite. The intensified interest in polymer nanocomposites with silver nanoparticle is due to the high antimicrobial effect of nanosilver as well as the unique characteristics of polymers which include their excellent structural uniformity, multivalency, high degree of branching, miscellaneous morphologies and architectures, and highly variable chemical composition [39]. The silver nanoparticle are readily form bonds with the fibers and produce the nano engineered coating of the material. The high surface area relative to the volume of particles increases their chemical reactivity, allowing them to stick to materials more permanently. The nanoengineered fabrics that kill bacteria, eliminate moisture, odor, and prevent static electricity. Polymer nanofiber coatings applied to textiles that make a bond to the fabric material at one end of the polymer fabrics. These polymer nanofiber coated on the surface of the materials prevent liquid contact or act as a waterproof to the textile materials. Nanofabrics with dirt-proof, stain-proof, and super hydrophobic properties are possible as a result of the layer formed by polymer nanofibers [40]. The Ag-fabric composite were prepared and experimented against Escherichia coli (gram-negative) and Staphylococcus aureus (gram-positive) cultures, showed stron antibacterial activity [41]. The electrospun nanocomposites of Poly vinyl alcohol (PVA) and Poly vinyl pyrrolidone (PVP) prepared by immobilizing the silver nanoparticle because of the strong affinity with a pyridyl group of metals and its ability to undergo hydrogen bonding with polar species [42].

VI. ENVIRONMENTAL APPLICATION OF WATER TREATMENT 


\section{USING SILVER NANOPARTICLES AND SILVER NANOCOMPOSITES}

Waterborne pathogens in drinking water makes health risk to the human beings as well as ecosystems. For instance, $\mathrm{Ag}$ and $\mathrm{Ag}$-containing compound nanomaterials, mostly in the form of nanoparticles and nanocomposites, are intensively used for diverse bio-related applications. Silver has also been impregnated in filters in point-of-use tap water purification devices in order to retard the growth of microorganisms. So, it can be deduced that the water purification filter which possesses Ag-containing nanomaterials can be a more costeffective way because of its semi-permanent characteristics than chemical method. The inhibitory effect of Ag+ ions on bacterial growth is due to their adsorption to negatively charged bacterial cell walls, inactivation of enzymes, disruption of membrane permeability, and, ultimately, cell death [43, 44]. Moreover, silver nanoparticle was reported as stable, biocompatible and non-toxic at lower concentration, the silver nanoparticle was not influencing the human epidermal karatinocytes [45]. The lower concentration of silver nanoparticle from 2 ppm -4 ppm was reported as nontoxic to human cells (HEK 293) and toxic to bacterial cells [46]. Silver nanoparticle can easily loaded within the porous substrates and the silver nanocomposites such as cellulose/Ag nanocomposites [47], Chitosan-silver nanocomposites [48], Silica silver composite [49] etc., are reported for its antibacterial characteristics and used in water treatment system. The Ag-NPs-porous ceramic composite was used as an antibacterial water filter. The thin-film nanocomposite ( $n$-TFN) membrane made up of multi-walled carbon nanotubes (MWNTs) and a thin-film layer containing nanosilver (nAg) particles are employed in water treatment system. Such silver nanoparticle loaded filters are potential against Pseudomonas aeruginosa PAO1, and proved as a efficient antibacterial agent [50]. The Sodium carboxymethyl cellulose (CMC) is the biocompatible and biodegradable polymer and are loaded with silver nanoparticle for water treatment application [51]. The silver nanoparticle, deposited on zeolite, sand, fibreglass, anion and cation resin substrates are used in ground water purification systems for the pathogenic bacterium removal [52]. The nanocomposite membranes incorporated with silver nanomaterials and titania nanomaterials exhibited good salt rejection capacity. Such effective membrane permeability and salt rejection of nanomaterials coated in a composite ceramic membranes and alumina ceramic membranes incorporated with silica nanomaterials are reported for their higher afficiency against salt rejection [53].

\section{CONCLUDING REMARKS}

The valuable properties of silver nanoparticle make them useful in diverse application. The silver nanoparticle composites also possess the significance and useful in the field of optical, electronic, electrical, medical and environmental applications. The antimicrobial nature of $\mathrm{AgNp}$ is very much useful in the field of medicine, paint, textile and environment. Silver nanoparticle are used as an important supplement for commercial products such as cosmetics, food industries etc.,. Future scope of silver nanoparticle in the field of advanced therapeutic, site directed treatment for cancer cells and fluorescence imaging of infected cells.

\section{Conflict of Interest}

The authors have declared no conflict of interest.

\section{REFERENCES}

[1] J. Toudert, H. Fernandez, D. Babonneaue, S. Camelio, T. Girardeau and J. Solis, Linear and third order non linear optical responses of multilayered $\mathrm{Ag}: \mathrm{Si}_{3} \mathrm{~N}_{4}$ Nanocomposites, Nanotechnology 20 (2009), pp. 475-705.

[2] C. Rao, G. Kulkarni, P. Thomas and P. Edwards, Size dependent chemistry; properties of nanocrystals, Chem. Eur.J. 8 (1) (2002), pp. 28-35.

[3] S. Bruzzone, M. Malvaldi, G. Arrighini and C. Guiditti, Theoritical study of electromagnetic scattering by metal nanoparticles, J. Phys. Chem. B. 109 (2005), pp. 3807-3812.

[4] H. Inouye, K. Tanaka, I. Tanahashi, T. Hattori and H. Nakatsuka, Ultrafast optical switching in a silver nanoparticle system, Jpn. J. Appl. Phys., 39 (2000), pp. 5132-5133.

[5] H. Park, R.P. VanDuyne and C.R. Yonzon, Optimizing the optical properties of functionalized silver nanoparticles by size, Spring Nanoscape 1(3) (2006), pp. 77-81.

[6] C. Wang, M. Luconi, A. Masi and L. Fernandez, Silver Nanoparticles as Optical Sensors, Silver Nanoparticles, David Pozo Perez (Ed.), InTech. (2010).

[7] F. Chen and R.L. Johnston, Plasmonic properties of silver nanoparticles on two substrates, plasmonics 4 (2009), pp. 147-152.

[8] A. Power, J. Cassidy and T. Betts, Non aggregated colloidal silver nanoparticle for surface enhanced raman spectroscopy, The Analyst 136 (2011) 2794-2801.

[9] A.Q. Wang, C.M. Chang and C.Y. Mou, Evolution of catalytic activity of Au-Ag bimetallic nanoparticles on mesoporous support for CO Oxidation, J. Phys. Chem. B. 109 (40) (2005), pp. 18860-18867. 
[10] O.M. Bakr, V. Amendola, C.M. Aikens, W. Wenseleers, R. Li, L.D. Negro, G.C. Skhatz and F. Stellacci, Silver nanoparticle with Broad Multiband Linear Optical Absorption, Angew. Chem. Int. Ed. 48 (2009), pp. 5921-5926.

[11] J. Jin, X. Ouyang, J. Li, J. Jiang, H. Wang, Y. Wang and R. Yang, Nucleic acid-modulated silver nanoparticles: A new electrochemical platform for sensing chloride ion, Analyst 136 (18) (2011), pp. 3629-34.

[12] E. Hutter, J.H. Fendler and D. Roy, Surface Plasmon Resonance Studies of Gold and silver nanoparticles linked to gold and silver substrates by 2-Aminoethanetiol and 1,6-Hexanedithiol, J. Phys. Chem. B. 105 (2011), pp. 11159-11168.

[13] F.J. Beck and K. Catchpole, Red shifting the surface plasmon of silver nanoparticles for light trapping in solar cells, MRS Proceedings, Cambridge Journals materials research society 2008.

[14] I. Prosycevas, J. Puiso, A. Guobiene, S. Tamulevicius and R. Naujokaitis, Investigation of Silver Polymer Nanocomposites Mater. Sci. 13 (3) (2007), pp. 188-192.

[15] J. Marques-Hueso, R. Abargues, J. Canet-Ferrer, J.L. Valdes and J. Martinez-Pastor, Resist-based silver nanocomposites synthesized by lithographic methods, Microelectronic Engineering 87 (2010), pp. 11471149.

[16] A. NimrodhAnanth, S. Umapathy, J. Sophia, T. Mathavan and D. Mangalaraj, On the optical and thermal properties of in situ/ex situ reduced Ag NP's/PVA composites and its role as a simple SPR-based protein sensor, Appl Nanosci. 1 (2011), pp. 87-96.

[17] H. Guo and S. Tao, Silver nanoparticles doped silica nanocomposites coated on an optical fiber for ammonia sensing, Sensors and Actuators B: Chemical (123)1 (2007), pp. 578-582.

[18] T. Endo, R. Ikeda, Y. Yanagida and T. Hatsuzawa, Stimuli-responsive hydrogel-silver nanoparticles composite for development of localized surface plasmon resonance-based optical biosensor, Aanalytica Chimica Acta (611) (2008), pp. 205-211.

[19] M. Umadevia and A. Jegatha Christya, Optical, structural and morphological properties of silver nanoparticles and its influence on the photocatalytic activity of $\mathrm{TiO}_{2}$, Spectrochimica Acta Part A: Molecular and Biomolecular Spectroscopy 111 (2013), pp. 80-85.

[20] D. Cheng, X. Qiao, X. Qin and J. Chen, Synthesis and electrical properties of uniform silver nanoparticles for electronic applications, J. Mater. Sci. 44 (2009), pp. 1076-1081.

[21] A.H. Alshehri, M. Jakubowska, A. Mlozniak, M. Horaczek, D. Rudka, C. Free, and J. DavidCarey, Enhanced Electrical Conductivity of Silver Nanoparticles for High Frequency Electronic Applications, ACS Appl. Mater. Interfaces 4 (2012), pp. 7007-7010.

[22] R. Chapman and P. Mulvaney, Electro-optical shifts in silver nanoparticle films, Chemical physics letters (349)5 (2001), pp. 358-362.

[23] H. Wei and H. Eilers, Electrical Conductivity of Thin-Film Composites Containing Silver Nanoparticles Embedded in a Dielectric Teflon ${ }^{\circledR}$ AF Matrix Eilers, Thin Solid Films 517 (2008), 575—581.

[24] Y. Oh, D. Suh, Y. Kim, E. Lee, J. Mok, J. Choi and S. Baik, Silver-plated carbon nanotubes for silver/conducting-polymer composites, Nanotechnol. 19 (2008), pp. $495602-495606$.

[25] I. Sur, D. Cam, M. Kahraman, A. Baysal and M. Culka, Interaction of multi-functional silver nanoparticles with living cells, Nanotechnology 21 (2010), pp. 175104.

[26] Y. Zhang, K. Zhang and H. Ma, Electrochemical DNA biosensor based on silver nanoparticle / poly(3-(3pyridyl acrylic acid)/ carbon nanotube modified electrode, Analytical biochemistry 387(1) (2009), pp. 1319.

[27] R.C. Pimentel, E.S.M. Martínez, A.M. Garcia, C.G. Garcia and Q.G.A. Palacios, Silver Nanoparticles Nanocarriers, Synthesis and Toxic Effect on Cervical Cancer Cell Lines, BioNanoScience 3(2) (2013), 198-207.

[28] S.H. Shin, M.K. Ye, H.S. Kim and H.S. Kang, The effects of nano-silver on the proliferation and cytokine expression by peripheral blood mononuclear cells, Int. Immunopharmacol. 7 (2007), pp. 18131818.

[29] J. Tian, K.K. Wong, C.M. Ho, C.N. Lok, W.Y. Yu, C.M. Che, J.F. Chiu and P.K. Tam, Tropical delivery of silver nanoparticles promotes wound healing, Chem. Med. Chem.. 2 (2007), pp. 129-136.

[30] C. Liu, X. Yang, H. Yuan, Z. Zhou and D. Xiao, Preparation of Silver Nanoparticle and Its Application to the Determination of $c t$-DNA, Sensors 7(2007), pp. 708-718.

[31] W. Wan, J.T.W. Yeow, M.I. Van Dyke, Effect of silver and titanium dioxide nanoparticles on PCR efficiency, Nanotechnology (2009) $9^{\text {th }}$ IEEE conference, pp. $458-461$.

[32] C. Caro, P.M. Castillo, R. Klippstein, D. Pozo and A.P. Zaderenko, Silver nanoparticles: sensing and imaging applications, David Pozo Perez (2010) InTech.

[33] J. Wu, S. Balasubramanian, D. Kagan, K.M. Manesh, S. Campuzano and J. Wang, Motion-based DNA detection using catalytic nanomotors, Nature Communications 1 (36) (2010), pp. 1-4. 
[34] K. Madhumathi, P.T. SudheeshKumar, S. Abhilash, V. Sreeja, H. Tamura, K. Manzoor, S.V. Nair, R. Jayakumar, Development of novel Chitin/Nanosilver Composite scaffolds for wound dressing applications, Journal of Material sciences materials in medicine 21 (2) 2010, pp. 807-813.

[35] Q. Ashton Acton, Advances in biomedical engineering research and application, Scholarly edition (2011), USA.

[36] V. Alt, T. Bechert, P. Steinrücke, M. Wagener, P. Seidel, E. Dingeldein, D. Scheddin, E. Domann and R. Schnettler, Nanoparticulate silver- A new antimicrobial substance for bone cement, Orthopade. 33 (2004), pp. 885-892.

[37] K.S. Morley, P.B. Webb, N.V. Tokareva, A.P. Krasnov, V.K. Popov, J. Zhang, C.J. Roberts and S.M. Howdle, Synthesis and characterisation of advanced UHMWPE/silver nanocomposites for biomedical applications, Eur. Polym. J. 43, (2007), pp. 307-314.

[38] M.S. Cohen, J.M. Stern, A.J. Vanni, R.S. Kelley, E. Baumgart, D. Field, J.A. Libertino and I.C. Summerhayes, In vitro analysis of a nanocrystalline silver-coated surgical mesh. Surg. Infect. 8 (2007), pp. 397-403.

[39] X. Xu, Q. Yang, Y. Wang, H. Yu, X. Chen and X. Jing, Biodegradable electrospun Poly (L-lactide)fibers containing antibacterial silver nanoparticles, European Polymer Journal 42 (2006), pp. 2081-2087.

[40] P. Dallas, V. K. Sharma and R. Zboril, Silver polymeric nanocomposites as advanced antimicrobial agents: Classification, synthetic paths, applications, and perspectives, Advances in Colloid and Interface Science (166) 1 (2011), pp. 119-135.

[41] I. Perelshtein, G. Applerot, N. Perkas, G. Guibert, S. Mikhailov and A. Gedanken, Sonochemical coating of silver nanoparticles on textile fabrics (nylon, polyester and cotton) and their antibacterial activity, Nanotechnology 19 (2008), pp. 245705.

[42] H. Niu, T. Lin and X. Wang, Needleless electrospinning. I. A comparison of cylinder and disk nozzles, J Appl Polym Sci. 114 (2009), pp. 3524-3530.

[43] J.M. Lagaron, M. Jose and A. Lopez-rubio, Antimicrobial Plastics Based on Metal-Containing Nanolayered Clays, John wiley \& sons, inc, Hoboken (2011) New Jersey, USA.

[44] V. Sambhy, M.M. MacBride, B.R. Peterson and A. Sen, Silver bromide nanoparticle/polymer composites: Dual action tunable antimicrobial materials, Journal of the American Chemical Society 128 (30) (2006), pp. 9798-9808.

[45] M. Rai and C. Posten, Green Biosynthesis of Nanoparticles Mechanisms and Applications, CABI Publishers (2013), UK.

[46] F. Okafor, A. Janen, T. Kukhtareva, V. Edwards and M. Curley, Green synthesis of silver nanoparticles, their characterization, application and antibacterial activity, International Journal of Environmental Research and Public Health 10 (2013), pp. 5221-5238.

[47] R.J.B. Pinto, P.A.A.P. Marques, C.P. Neto, T. Trindade, S. Daina and P. Sadocco, Antibacterial activity of nanocomposites of silver and bacterial or vegetable cellulosic fibers, Acta Biomaterialia (5)6 (2009), pp. 2279-2289.

[48] K. Vimala, Y.M. Mohan, K.S. Sivudu, K. Varaprasad, S. Ravindra, N. Narayana Reddy, Y. Padma, B. Sreedhar and K. MohanaRaju, Fabrication of porous chitosan films impregnated with silver nanoparticles: A facile approach for superior antibacterial application, Colloids and Surfaces B: Biointerfaces 76 (2010), pp. 258-258.

[49] S. Egger, R.P. Lehmann, M.J. Height, M.J. Loessner and M. Schuppler, Antimicrobial Properties of a Novel Silver-Silica Nanocomposite Material, Applied and Environmental Microbiology, (75) 9 (2009), pp. 2973-2976.

[50] E.S. Kim, G. Hwang, M.G. El-Din and Y. Liu, Development of nanosilver and multi-walled carbon nanotubes thin-film nanocomposite membrane for enhanced water treatment, Journal of membrane science 394 (2012), pp. 37-48.

[51] A. Hebeish, M. Hashem, M.M. Abd El-Hady and S. Sharaf, Development of CMC hydrogels loaded with silver nano-particles for medical applications, Carbohydrate Polymers 92 (2013), pp. 407- 413.

[52] L. Mpenyana-Monyatsi, N.H. Mthombeni, M.S. Onyango and M.N.B. Momba, Cost-Effective Filter Materials Coated with Silver Nanoparticles for the Removal of Pathogenic Bacteria in Groundwater, Int. J. Environ. Res. Public Health 9 (2012), pp. 244-271.

[53] D.G. Kim, H. Kang, S. Han and J.C. Lee, The increase of antifouling properties of ultrafiltration membrane coated by star-shaped polymers, Journal of Material Chemistry 22 (2012), pp. 8654-8661. 